\title{
RHYTHMS IN THE REPRODUCTIVE ACTIVITY OF INFUSORIA
}

\author{
LORANDE LOSS WOODRUFF AND GEORGE ALFRED BAITSELL \\ Sheffeld Biological Laboratory, Yale University \\ THIRTEEN FIGURES
}

In a study of the life history of Paramaecium caudatum by pedigree cultures, Calkins clearly illustrated the cycle, while the rhythms in the division rate were later emphasized by Woodruff in a study of the life history of several species of hypotrichous Infusoria. The fluctuations in their rate of reproduction were classified as follows: ${ }^{1}$

"A rhythm is a minor periodic rise and fall of the fission rate, due to some unknown factor in cell metabolism, from which recovery is autonomous."

A cycle is a periodic rise and fall of the fission rate, extending over a varying number of rhythms, and ending in the extinction of the race unless it is 'rejuvenated' by conjugation or changed environment" (cf. fig. 1).

Gregory, in a study of the life history of Tillina magna, stated that "The curve which represents the general vitality of the protoplasm shows the normal rhythmic fluctuations observed by Woodruff." Gregory also made an analysis of the data secured by Popoff in his study of the life history of Stylonychia mytilus, and she stated that "If the curve of Stylonychia is plotted from average records of five and ten day periods, it will be found to correspond to the curves of Paramaecium, Oxytricha and Tillina, each showing the rhythmic periods of high and low vitality." More recent work has shown that Paramaecium aurelia may be bred indefinitely on a culture medium which is varied from day to day, ${ }^{2}$ i.e., the cycle does not occur under these conditions though

${ }^{1}$ Woodruff ('05). $\quad{ }^{2}$ Cf. Woodruff ('11a), Taf. 26, 27.

THE JOURNAL OF EXPERIMENTAL ZOÖLOGY, VOL. 11, No. 4

NOVEMBER, 1911 


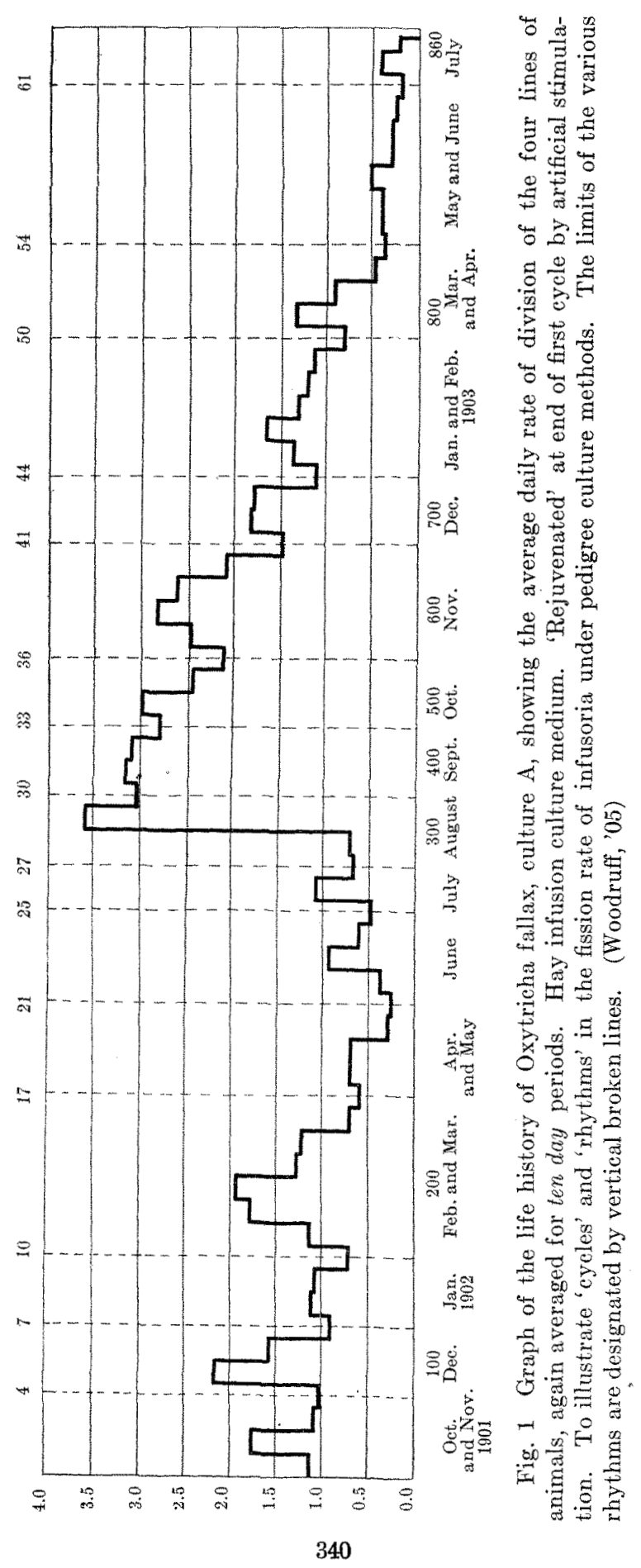




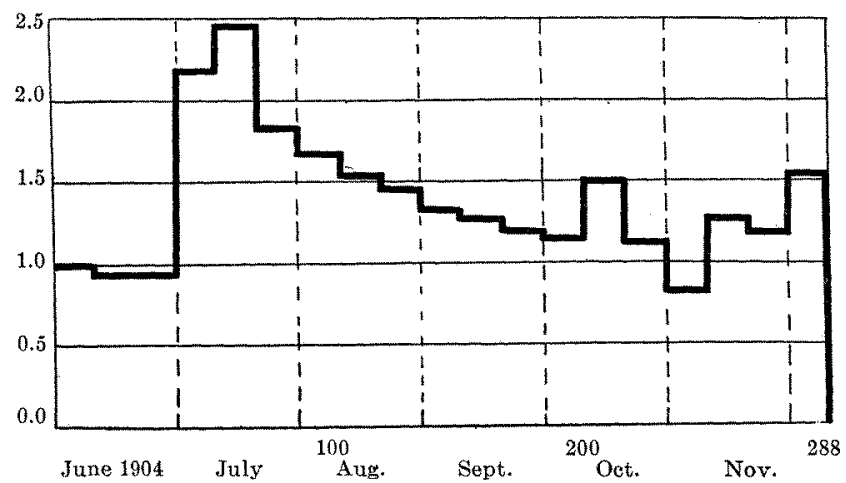

Fig. 2 Graph of the life history of Gastrostyla steinii showing the average daily rate of division of the four lines of animals which compose the culture, again averaged for ten day periods. Hay infusion culture medium. To illustrate a case where the rhythms are apparently absent for a considerable period. Compare with fig. 3. (Woodruff, '05)

the rhythms persist undiminished; and also that the same result may be attained by a constant culture medium of beef extract ${ }^{3}$ (cf. fig. 4).

It is obvious from these investigations that the life history of Infusoria in pedigree cultures comprises many minor rhythmic fluctuations in the fission rate from which recovery is autonomous. The results with beef extract as a constant medium for Paramaecium aurelia naturally led to an intensive study of therhythms, in order to determine if these also can be eliminated by a still more constant environment, i.e., whether they are due to minor variations in the environment or to unknown intracellular phenomena, as originally stated. Possible sources of variation in the environment which might give rise to variations in the metabolism of the cell which would become apparent as rhythms in the rate of reproduction are: 1) Chemical composition of the culture medium, 2) Quantity and quality of the bacterial flora of the culture medium, 3) Excretion products of the paramaecia, 4) Mechanical stimulation during isolation, 5) Light, 6) Barometric pressure, and 7) Temperature. The data secured which bear on this question are given in the present paper.

${ }^{3}$ Woodruff' and Baitsell ('11). 


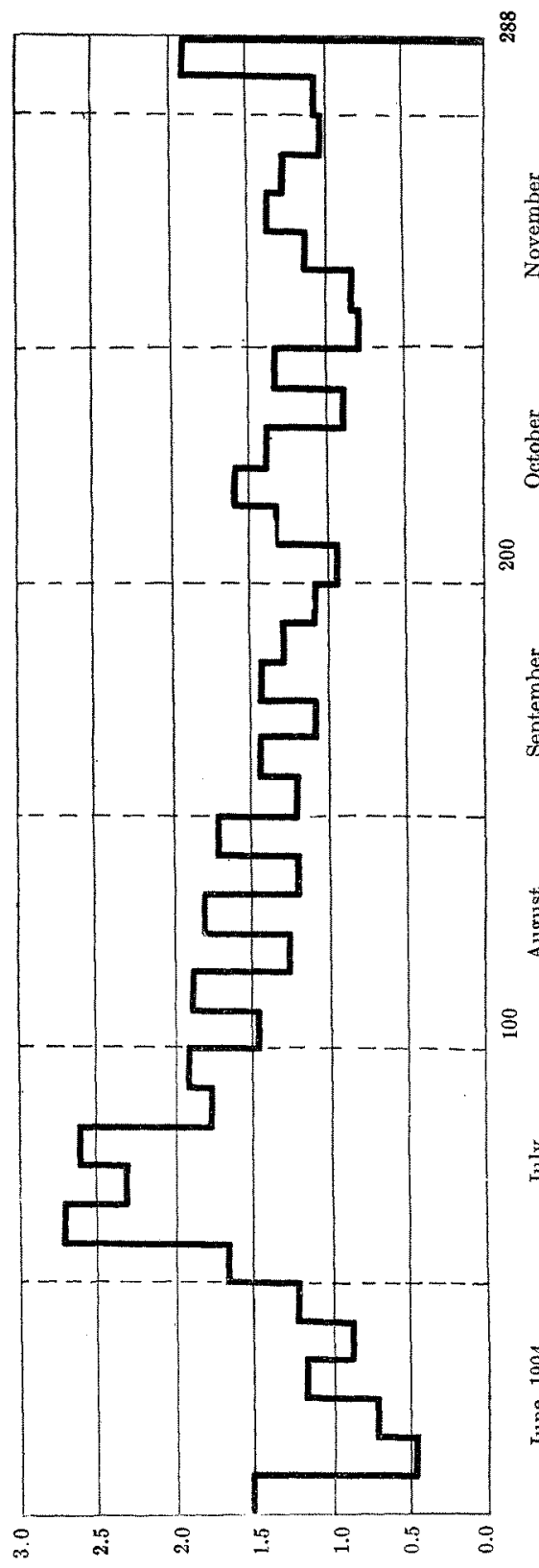

密总总

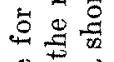

궁

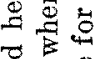

$\stackrel{5}{8} 80$

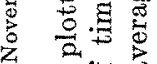

㟧

용

家定

oi $\stackrel{5}{3}$

草

○.

$5 \div \frac{15}{50}$

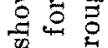
: 7 霜 용 证 $\stackrel{\overrightarrow{0}}{\circ}$ ${ }_{0} \rightarrow$ है ए है प홍

范要密

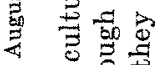
है 氶

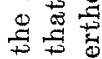
$\circlearrowleft \circlearrowleft$

$\exists$ ड़ष्ठ

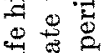

녕

+

兽 运

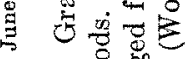
क官焉

$s_{1}=8$

焉. 


\section{METHODS}

The animals employed in this study were taken from the pedigree culture of Paramaecium aurelia (I) which one of us $^{4}$ has had under daily observation for fifty-one months and which has attained 2500 generations, up to the present time (August 1, 1911), under the conditions of a varied environment, without conjugation or artificial stimulation. From this culture a subculture was isolated line by line on October 1, 1910, at the 2012th generation, and carried for ten months on a constant culture medium of beef extract. It was then discontinued. ${ }^{5}$ The average daily rate of division of the four lines of this subculture (IB), again averaged for five day periods, was computed and the result is graphically shown in fig. 4.

The experiments in regard to the rhythms were begun on June 8,1911 , by isolating two subcultures line by line from IB at the 2335th generation, and placing the animals in a similar manner on depression slides in five drops of the beef extract medium. This medium consisted of a 0.025 per cent solution of Liebig's extract of beef. The slides were kept in small moist chambers to prevent evaporation. The cultures were continued by isolating each day an organism from each of the four lines of the respective cultures, and placing it in fresh medium on a sterile depression slide. The number of divisions during the previous twenty-four hours was recorded at the time of isolation and from this data the graphs were drawn. One of these two subcultures was placed in a thermostat chamber at a temperature of practically $82^{\circ} \mathrm{F}$. (culture IB82a) and the other in a chamber at a temperature of practically $76^{\circ} \mathrm{F}$. (culture IB76a), and maintained at this temperature for forty days.

A second series of two subcultures was similarly started from IB on June 18th, at the 2346th generation, and treated exactly the same as the above cultures. The cultures of this series were designated IB82b and IB76b, respectively. A third series of two subcultures was isolated in the same manner from IB on June 28 th, at the 2355th generation, and these cultures were

${ }^{4}$ Woodruff ('11a).

${ }^{5}$ For further details of this subculture (IB) ef. Woodruff and Baitsell ('11). 
344 LORANDE L. WOODRUFF AND GEORGE A. BAITSELL

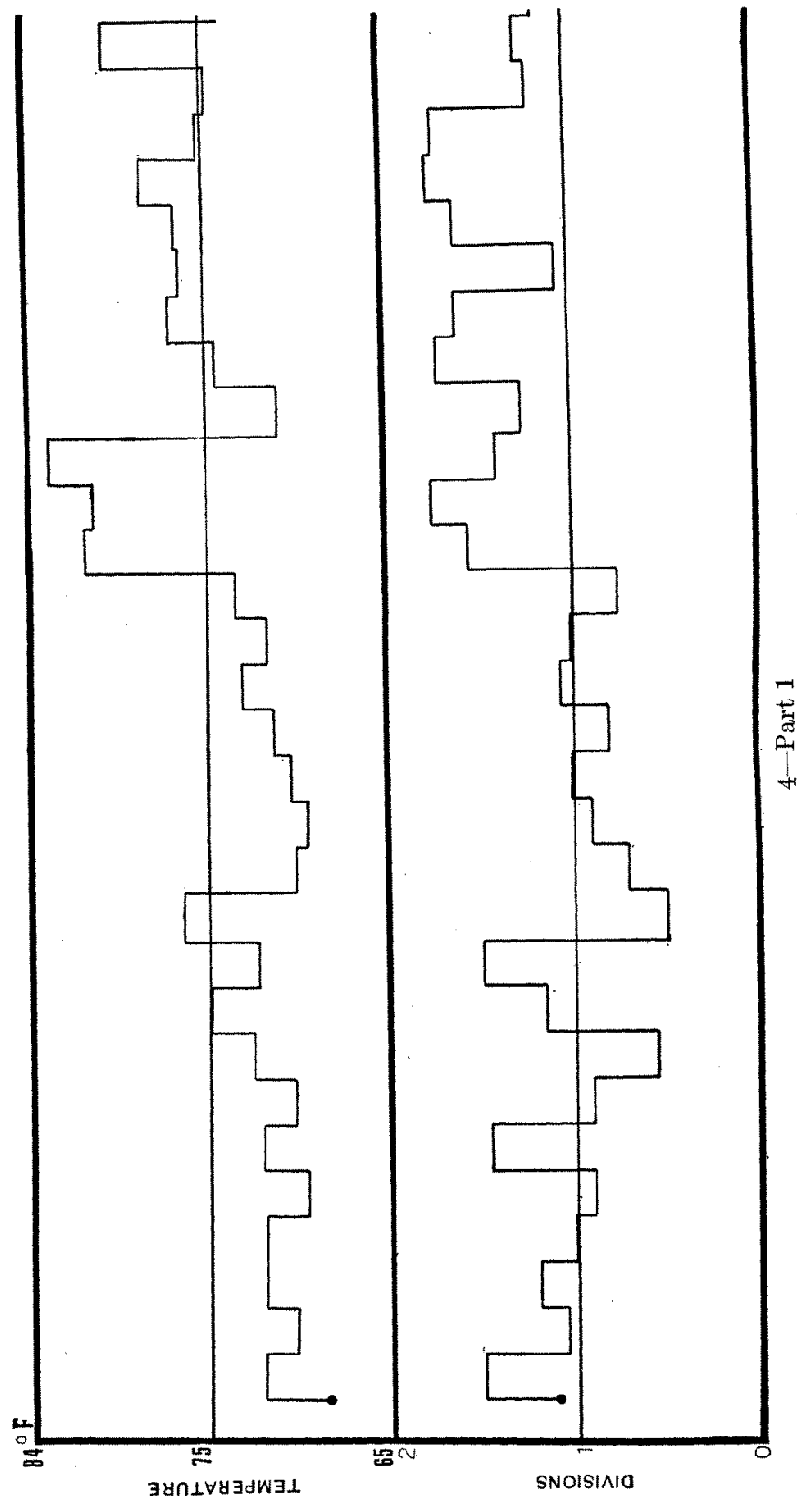




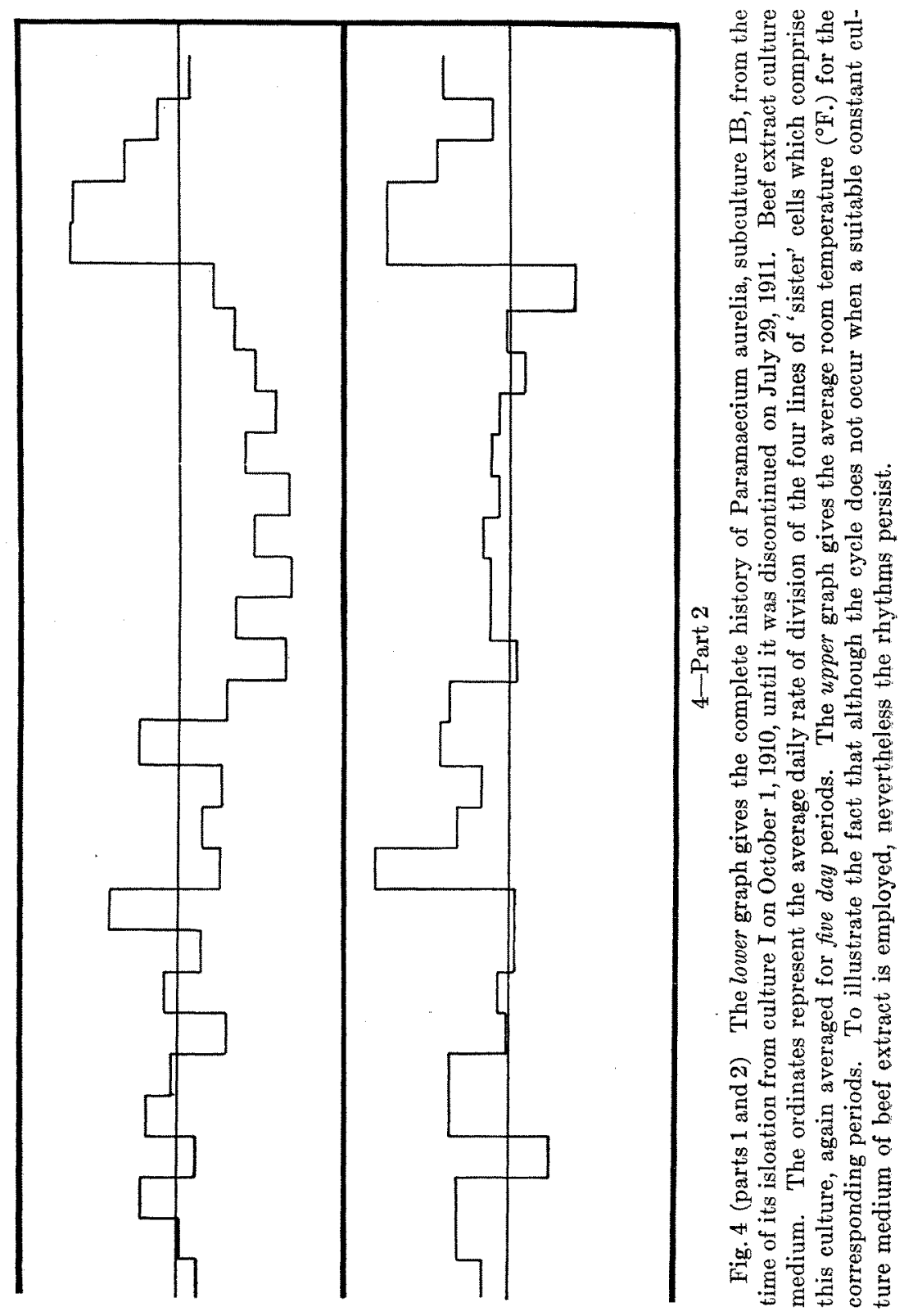


designated IB82c and IB76c. There were then the following cultures, all of which were kept in the dark, involved in this experiment:

IB-at room temperature.
IB $82 \mathrm{a}$-isolated from IB and continued for forty days at $82^{\circ} \mathrm{F}$.
IB $76 \mathrm{a}$-isolated from IB and continued for forty days at $76^{\circ} \mathrm{F}$.
IB82b-isolated from IB and continued for forty days at $82^{\circ} \mathrm{F}$.
IB $76 \mathrm{~b}$-isolated from IB and continued for forty days at $76^{\circ} \mathrm{F}$.
IB82c-isolated from IB and continued for thirty days at $82^{\circ} \mathrm{F}$.
IB $76 \mathrm{c}$-isolated from IB and continued for thirty days at $76^{\circ} \mathrm{F}$.

Since the entire point involved in this study depends upon the constancy of the environment to which the animals are subjected, this will be considered in detail.

1. Chemical composition of the culture medium. The culture medium was made up by weighing out the proper amount of Liebig's extract of beef and diluting it with distilled water. The solution was then put into about one hundred test tubes, plugged with cotton and sterilized. The medium remained sterile until used. Since all the culture medium which was used throughout the experiment was made up at one time there was no variation in the medium itself during the work.

2. Quantity and quality of the bacterial flora of the culture medium. Paramaecium is an animal which depends on bacteria for its food, and consequently these must be supplied. Sufficient bacteria were 'automatically' transferred with the animals at the first isolation to provide ample food until the next isolation at the end of twenty-four hours. Again at this time sufficient bacteria were 'automatically' carried over. with the animals to infect the fresh medium in which they live for the following twenty-four hours, and so on. The quality of the bacterial flora was initially the same on all the slides because all the paramaecia used to start the various lines were taken from the same environment when the experiments were begun, and it is believed that this condition was maintained by the cross infection of all the slides almost daily. This also served to eliminate variations due to infections from the air of the moist chambers. Obviously the number of bacteria on a slide varied during the twenty-four hours between isolations. 
But a study of the preparations showed that the paramaecia keep down the results of the rapid multiplication of the bacteria by feeding on them, so that, although there is ample food for the animals at all times, the variation in the bacterial content of the medium is not so great as would at first glance appear to be the case. However, the point to be emphasized is that these variations, small as they were, were only of twenty-four hour duration since fresh culture medium was supplied daily. Consequently any effect of the slight and unavoidable variation in the quantity of the bacteria could result only in an intradiurnal rhythm in the division rate, and since the count of the generations was taken at twenty-four hour intervals, this variation would not appear in our records. Elaborateness of method is not necessarily coincident with exactness of technique, and therefore it was considered unnecessary to attempt to 'sterilize' the paramaecia and feed them on pure cultures of bacteria. Any effort in this direction has met only with partial success and has introduced complicating factors which, it is believed, would more than counterbalance any advantages to be gained for the problem in hand.

3. Light. Throughout the experiments all the cultures were in absolute darkness except for the short time daily when the count was being made. This was unavoidable, but each animal was not exposed to the light for more than three minutes. A control culture carried in the light showed that light does not influence the rate of reproduction of paramaecium. This is in accord with the previous results ${ }^{6}$ on the effect of light on the division rate of free-living Infusoria.

4 and 5. Excretion products of paramaecia and mechanical stimulation during isolation. These factors may be eliminated because they could only give rise to an intradiurnal rhythm which would not appear in the data.

6. Barometric pressure. A careful study was made of the variations in the barometric pressure which occurred during the experiments. There was absolutely no correlation between the small fluctuations in pressure and the rhythms in the division rate, and

'Maupas ('88) and Woodruff ('05). 
consequently it can be positively stated that this factor plays no part in our results.

7 . Temperature. This is the chief possible variable in the environment which we have to consider. In the original discussion of rhythms it was stated that "the results serve to emphasize the fact that while temperature does influence the rate of multiplication, it is not the most important element among the factors which cause fluctuations." A study of figs. 4 and 5 shows that there is a certain amount of correlation between the fluctuations of fission rate and of temperature, when the cultures aresubjected to the ordinary changes in temperature of the laboratory.

Our experiments were carried on in a Panum thermostat, ${ }^{7}$ heated at one end with a gas flame (with an automatic regulator) and cooled at the other by a large ice chest. The thermostat was divided into nine chambers, grading down in temperature from one end to the other of the apparatus. The temperature was recorded in each chamber by a maximum and minimum registering thermometer, by a tube thermometer, and in one chamber also by a thermograph. Experiments were conducted in six of the nine compartments, but an account is given here of the results of the cultures at the two temperatures within the optimum zone for the strain of Paramaecium being used. The detailed data in regard to the effect of different temperatures on the division rate of this animal and its relation to the temperature coefficient of chemical reactions in general will be published later. We should state, however, that our results at other temperatures are entirely concordant with those here described.

The temperature selected for the study of the influence of temperature on the rhythms were approximately $82^{\circ} \mathrm{F}$. and $76^{\circ} \mathrm{F}$. as it was found that the optimum zone for the culture included these points. During the fifty days that the experiments covered the variations in temperature did not exceed $3^{\circ} \mathrm{F}$.; for the greater part of the time the variation was less than $1^{\circ} \mathrm{F}$, and for several periods it was less than $0.5^{\circ} \mathrm{F}$. The greatest variation noted

\footnotetext{
7 This apparatus was constructed for this and similar studies from an improved model designed by Professor L. F. Rettger of the Sheffield Bacteriological Laboratory of Yale University. Our thanks are due Dr. Rettger for his kind coöperation.
} 


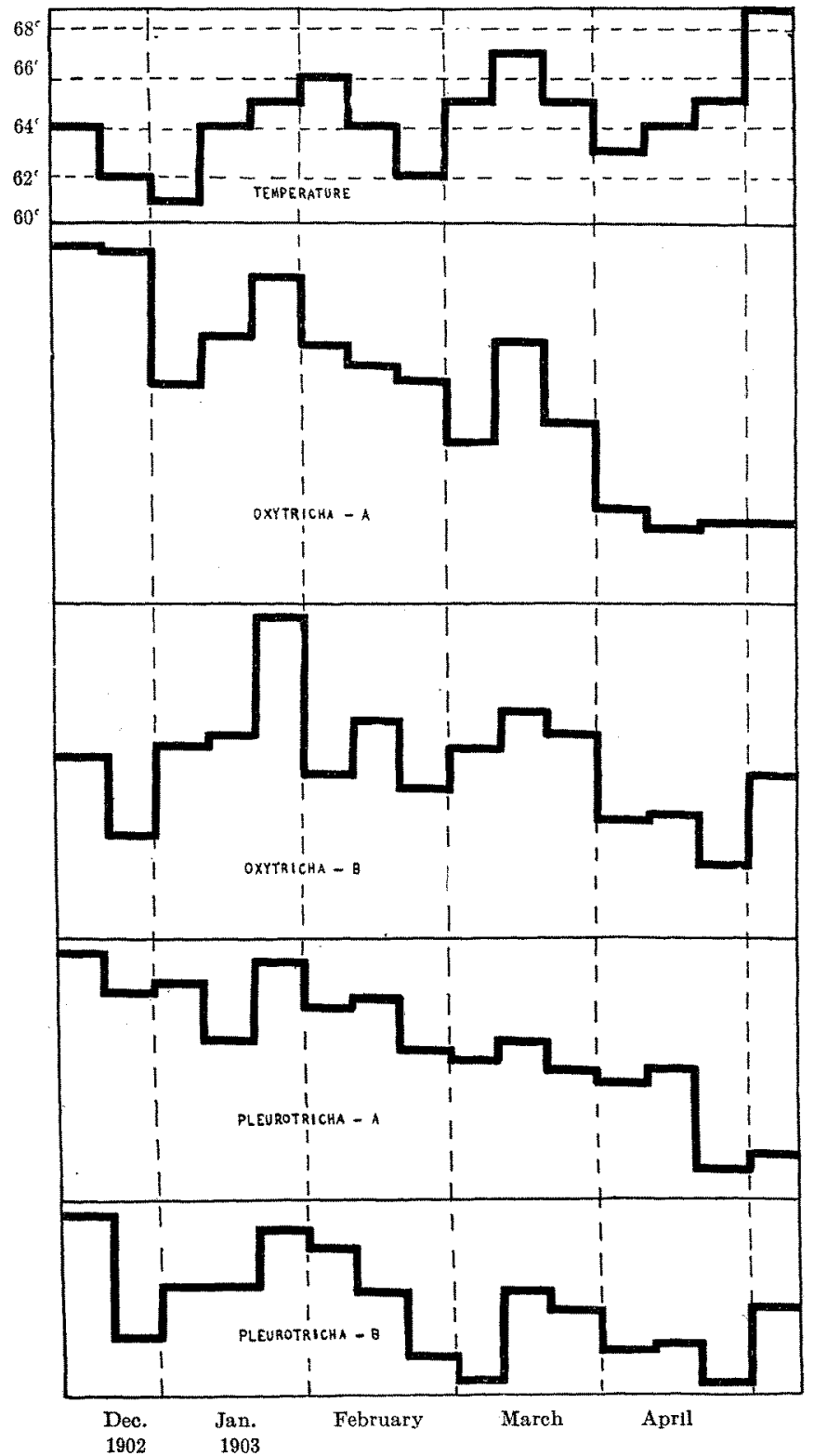

Fig. 5 Sections of the culture graphs of two cultures of Oxytricha fallax and two cultures of Pleurotricha lanceolata, together with the graph of the average room temperature for the same period. Averages for ten day periods. To illustrate a striking instance of the apparent relation of rhythms to the fluctuations in temperature. (Woodruff, '05) 
above occurred during a week of unusually hot weather when the sudden change was too great to be immediately compensated for by the automatic regulator. Great care was taken in removing the preparations from the chambers for the daily count and isolation. The culture medium to be used on one day had been put the day before in the proper chamber of the thermostat, and consequently the animals were transferred to fresh culture medium of the same temperature. Of course any effect of variations arising from the daily transfers could only be intradiurnal and consequently would not appear in our results. It should also be emphasized that the recorded temperature was that of the air in the thermostat, whereas the animals were in culture fluid on slides within the moist chambers in the thermostat. The temperature of the moist chambers obviously was still more constant than that of the thermostat chamber, as likewise was the liquid in which the organisms were living. Consequently the variations in temperature which the animals experienced certainly never exceeded $3^{\circ} \mathrm{F}$. throughout the experiments, and this maximum variation occurred only at one period. For a period of ten consecutive days there was no visible variation greater than $0.4^{\circ} \mathrm{F}$. It is believed that the temperature conditions were maintained as nearly constant as modern apparatus and the necessities of the experiment allowed.

\section{RESULTS}

The results can be stated briefly because graphs of the rate of reproduction bring out the points involved far better than a description by words. Fig. $6, A$, gives the average daily rate of division of the four lines of 'sister' cells of Paramaecium aurelia, series IB82a, again averaged for ten day periods, at $82^{\circ} \mathrm{F} . \quad B$ and $C$ show the same for series IB82b and IB82c. Fig. 7, $A, B$, and $C$, shows similarly the results derived from IB76a, and IB76b, and IB76c. Figs. 8 and 9 give the same data averaged for five day periods. Fig. 10 shows the average daily rate of division, for five day periods, of line 1 (of the four lines) of series IB82a and line 1 of IB76a. Fig. 11 gives the same data for series IB82b and IB76b. Fig. 12 presents the average daily rate of division 


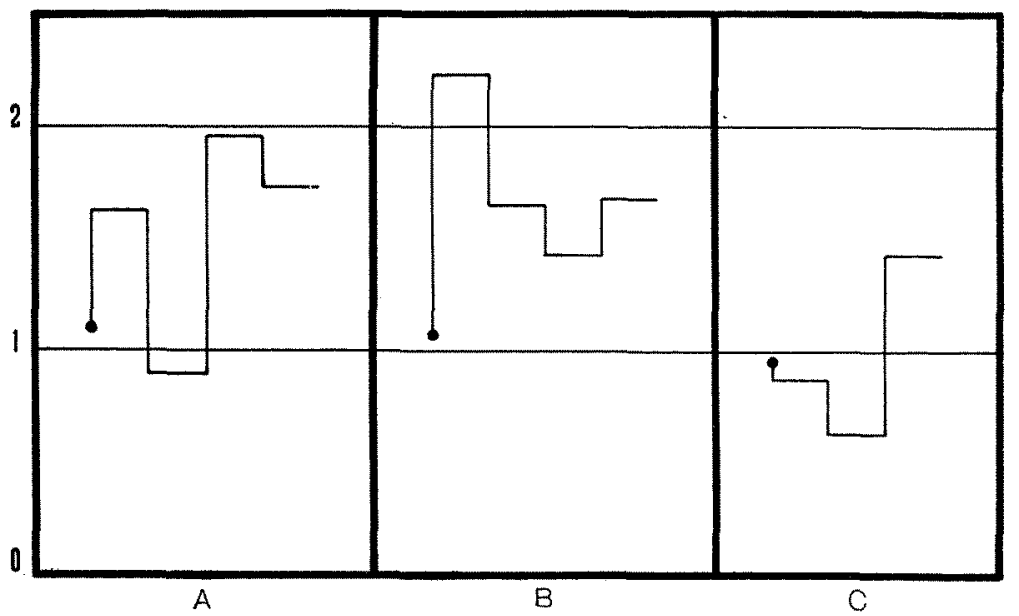

Fig. $6 A$, Graph of the average daily rate of division at $82^{\circ} \mathrm{F}$. of the four lines of 'sister' cells of Paramaecium aurelia, series IB82a, again average for ten day periods. $B$ and $C$, Similar graphs for series IB $82 \mathrm{~b}$ and IB $82 \mathrm{c}$ respectively. To illustrate rhythms in the fission rate when the cultures are subjected to practically constant conditions, including temperature.

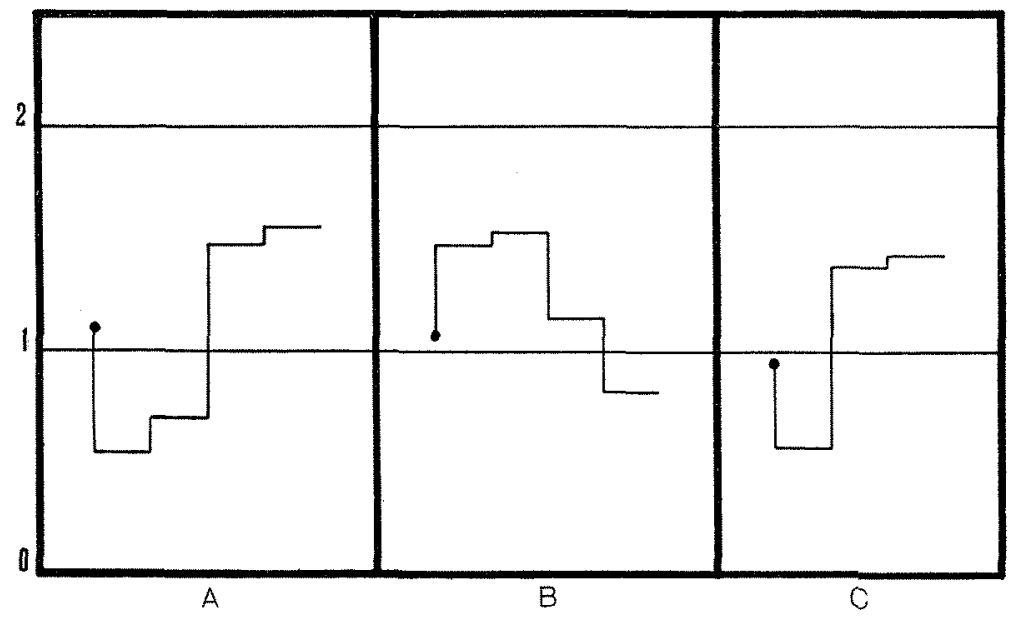

Fig. $7 A$, Graph of the average daily rate of division at $76^{\circ} \mathrm{F}$. of the four lines of 'sister' cells of Paramaecium aurelia, series IB76a, again averaged for ten day periods. $B$ and $C$, Similar graphs for series IB76b and IB76c respectively. To illustrate rhythms in the fission rate when the cultures are subjected to practically constant culture conditions, including temperature. Compare with fig. 6 . 


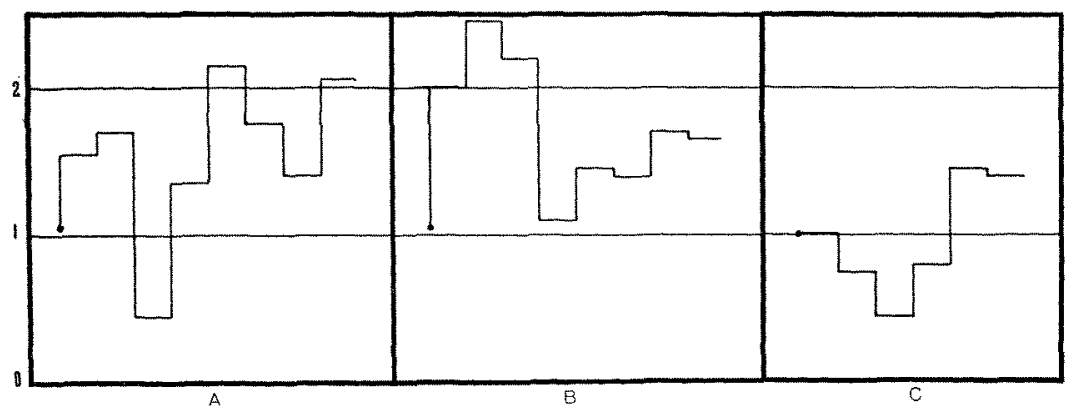

Fig. 8 A, Graph of the average daily rate of division at $82^{\circ} \mathrm{F}$. of the four lines of 'sister' cells of Paramaecium aurelia, series IB82a, again averaged for five day periods. $B$ and $C$., Similar graphs for series IB82b and IB82c respectively. To illustrate the fact that rhythms in the rate of division appear more pronounced under practically constant environmental conditions. Compare with the last ten periods of the culture subjected to room temperature changes (fig. 4).

of the four lines of IB82a and IB76a, respectively. The vertical dotted lines include the ten day period during which temperature variations were entirely absent, or not greater than $0.4^{\circ} \mathrm{F}$. Fig. 13 gives the same results for series IB82b and IB76b during the ten days of most constant temperature.

A study of these graphs of the rate of reproduction of Paramaecium shows that the exceptionally and practically constant conditions of the environment failed to diminish or eliminate the rhythms-but on the contrary tended to bring them out more clearly. The fact that the rhythms appear more pronounced

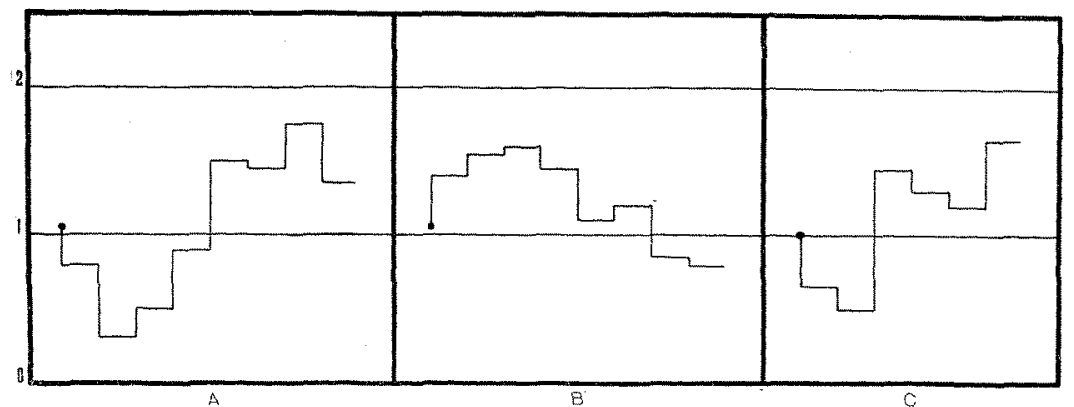

Fig. $9 A$, Graph of the average daily rate of division at $76^{\circ} \mathrm{F}$. of the four lines of 'sister' cells of Paramaecium aurelia, series IB76a, again averaged for five day periods. $B$ and $C$., Similar graphs for series $I B 76 \mathrm{~b}$ and $\mathrm{IB} 76 \mathrm{c}$ respectively. To illustrate the same point as fig. 8 . 


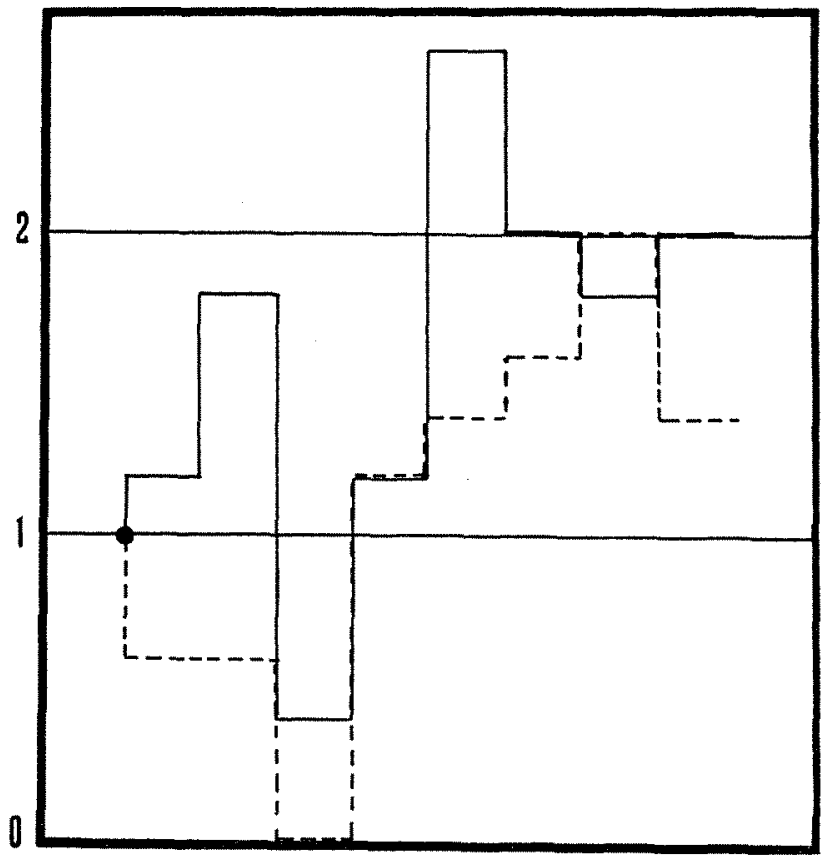

Fig. 10 Graph of the average daily rate of division for five day periods of line 1 (of the four lines of 'sister' cells) of series 1B82a (= continuous line) and of line 1 (of the four lines of 'sister' cells) of series IB76a (= . - line). To illustrate the fact that rhythms of practically the same amplitude and character appear in a graph of a single line of cells as appear when four such lines are averaged together. Compare with fig. 8, section A, and fig. 9, section A.

under the practically constant conditions existing during these experiments than they do under ordinary laboratory conditions, clearly suggests that they are due to a fundamental factor in cell phenomena and not to extraneous causes. For if they are due to inherent intracellular conditions, one would a priori expect to find them more clearly brought out when the cell is free from extraneous influences.

A study of the curves of the division rate at the two temperatures shows that temperature, as is well known, markedly influences the rate, but it also shows that the rhythms persist-the reproductive activity being, as it were, pitched at a higher scale, but its character in no wise altered. In other words, it is not sug- 


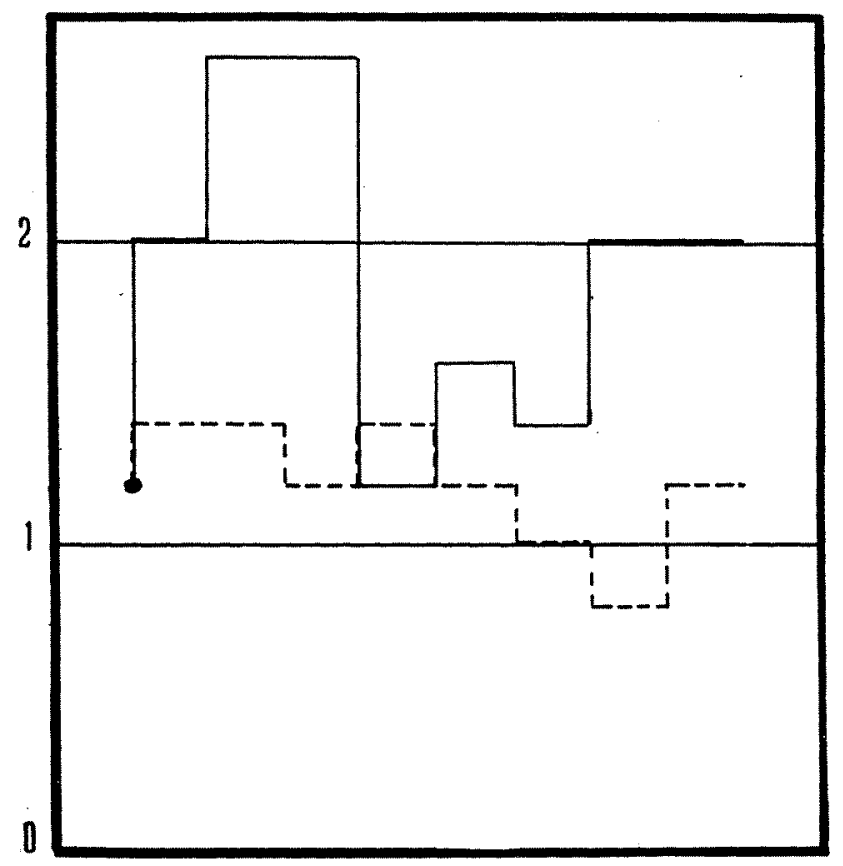

Fig. 11 Graph of the average daily rate of division for five day periods of line 1 (of the four lines of 'sister' cells) of series IB82b (= continuous line) and of line 1 (of the four lines of 'sister' eells) of series IB76b (= - - line). To illustrate the same points as fig, 10. Compare with fig. 8, section B, and fig. 9 , section B.

gested that the division rate is not largely a function of temperature-all other conditions being equal. It is probable that the temperature coefficient of the mean rate of division for a period including several rhythms will coincide closely with that of chemical reactions in general, but it is also probable for example that the rate of division at the crest of a rhythm at a high temperature and at the bottom of a rhythm at a low temperature will give a coefficient higher than the theory demands. Experiments to determine this point are in progress.

It should also be pointed out that the total number of divisions attained during a prolonged period of time is comparatively constant. For example, the number of generations attained by culture I during 1909 was 613 and during 1910 was 612 . Of 


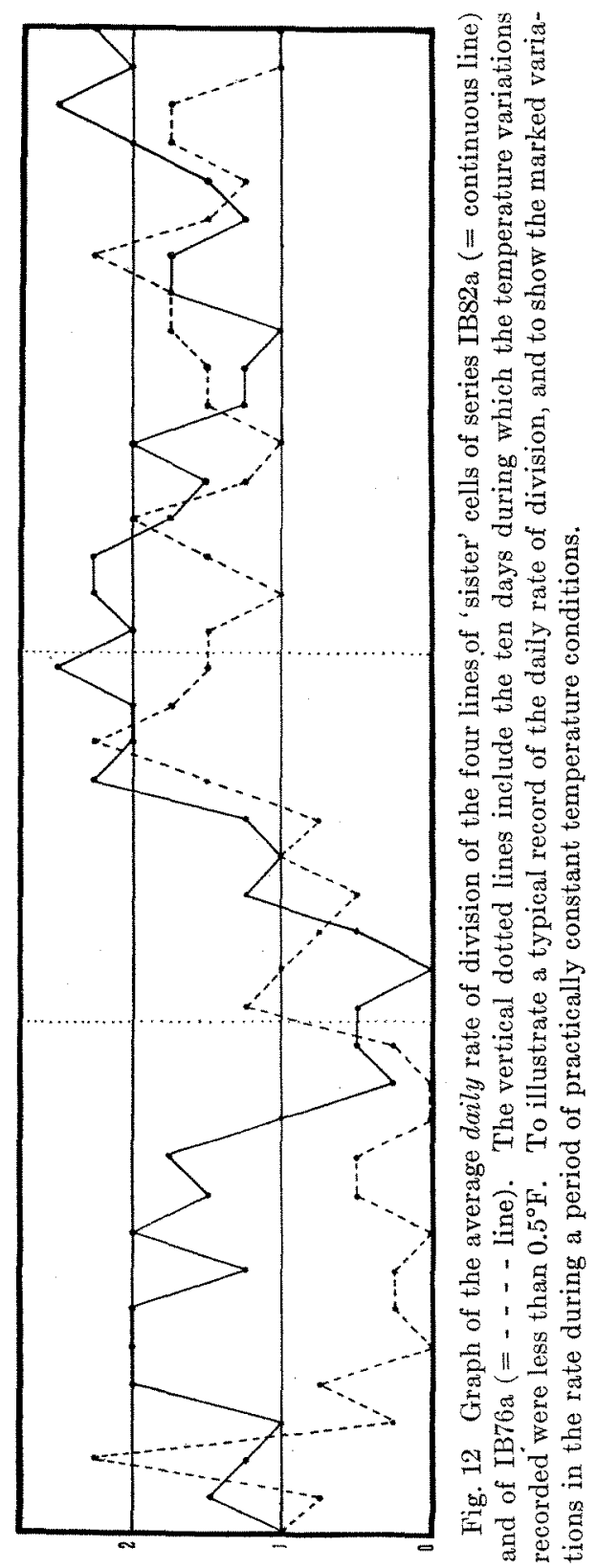

THE JOURNAL OF EXPERMMENAL ZOÖLOY, VOL, 11, NO. 4 


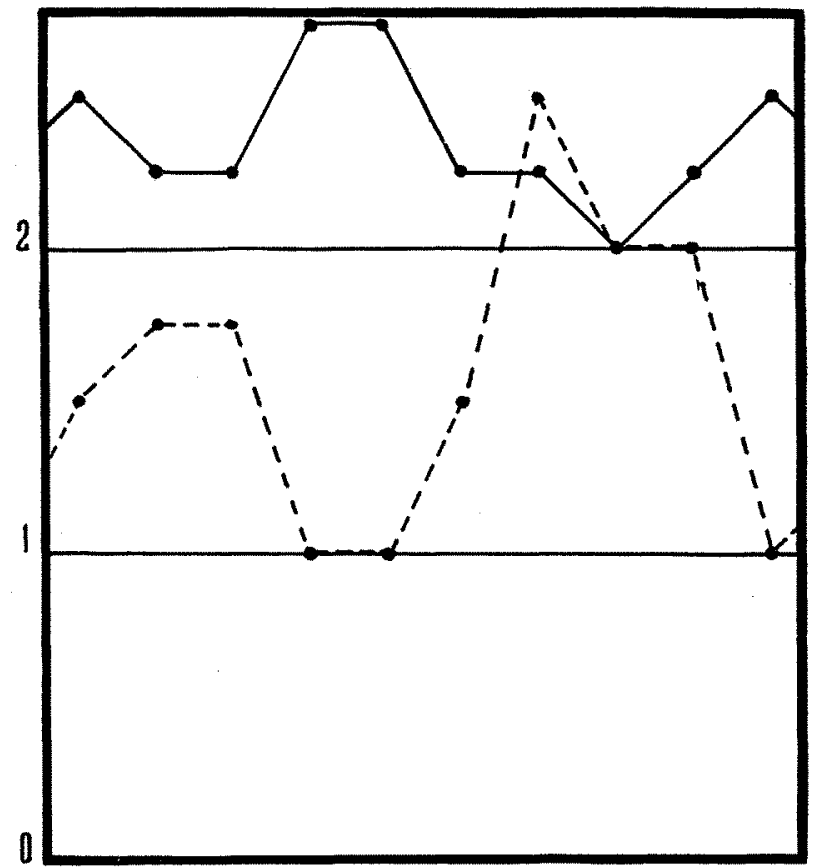

Fig. 13 Graph of the average daily rate of division of the four lines of 'sister cells of series IB $82 \mathrm{~b}$ ( $=$ continuous line) and of IB76b ( = - - line) for the ten days during which temperature variations were practically absent. To illustrate the same points as fig. 12. Compare with the section of fig. 12 included within the vertical dotted lines.

course this very exact coincidence is an 'accident' but, taken with a considerable amount of data along the same line, it quite definitely points to the fact that the organism has the potential for about a certain number of bipartitions during a long period of time and this number is approximately attained irrespective of the minor fluctuations in the rate, due to external or internal causes.

In a recent paper, Jennings states that

"Within the same line the rate is sometimes very different for a certain period, as a week or ten days, from the rate during the rest of the time. This is much more evident when one inspects a table in which the fissions are recorded day by day. The rate in a given line is there seen at times to drop, remain low for perhaps ten days, then return to the 
original rate. In most of all these cases there are evidences of pathological conditions during these periods of lowered rate of fission. Monstrosities appear, and many of the specimens die. Therefore these periods of slower rate are not to be considered as giving the characteristic rate for the race when healthy. In comparing different races, the periods when the rate of fission is high and uniform should be compared."

These observations of Jennings are difficult to understand in view of our results with Paramaecium. His statement in regard to weekly variations we would, at first glance, interpret as further evidence of rhythms; but throughout the more than four years of the life of this pedigree culture, a monster has never been seen in any of the direct lines, and only two or three times has a single deformed individual been seen in the heavy stock cultures which have been seeded from this strain. Further, it is an unusual occurrence for a line in any of our experiments to die out without assignable cause. Therefore it is necessary to emphasize that whereas the statement quoted seems, at first thought, to be in regard to periodic fluctuations in the rate of bipartition identical with those we call rhythms, nevertheless these fluctuations have absolutely nothing in common since, according to Jennings' statement, those occurring in his lines are pathological.

\section{CONCLUSIONS}

The results of studies on the life history of free living Infusoria by exact pedigree culture methods show that, when these organisms are bred on comparatively constant culture media of hay or other infusions, the reproductive activity shows cycles and rhythms. Further results show that when Paramaecium aurelia is bred on a varied culture medium, or on a constant medium of beef extract, cycles do not occur, but rhythms persist. The results given in the present paper show that it is not possible by constant environmental conditions to eliminate the rhythms and to resolve the graph of the multiplication rate into an approximately straight line. It therefore seems justifiable to conclude that there are inherent rhythmical changes in the phenomena of the cell which are brought to view still more clearly when not 
influenced by external factors. Variations in the rhythm of division is well-known in the development of the metazoon egg and it has yet to be satisfactorily explained. Towle in a paper on the effects of stimuli on Paramaecium makes the following statement: "There may even prove to be rythmical changes in sensitiveness like those described by Lyon for cleaving eggs, and Scott for unfertilized eggs. Something of this nature is indicated by the fact that paramaecia from the same culture vary in sensitiveness from day to day." Woodruff ('05) wrote: "In my work on the effect of chemicals on Infusoria I have found that individuals react differently at various times to a given stimulus and I believe we have the clue to these changes in sensitiveness manifested in the rhythms of the fission rate."

Finally, the data justify the conclusion that the cells of this pedigree culture of Paramaecium aurelia have the potentiality to perpetuate themselves indefinitely by division (under proper environmental conditions) - the only necessary variations in the rate of reproduction being normal minor periodic rises and falls of the fission rate, due to some unknown factor in cell phenomena, from which recovery is autonomous.

\section{LITERATURE CITED}

G. N. Calkins 1904 Studies on the life history of Protozoa. IV. Death of the A series. Conclusions. Journ. Exper. Zool., vol. 1, no. 3.

L. H. Gregory 1909 Observations on the life history of Tillina magna. Journ. Exper. Zool., vol. 6, no. 3.

H. S. Jennings and G. T. HargitT 1910 Characteristics of the diverse races of Paramaecium. Journ. Morph., vol. 21, no. 4.

E. P. LYoN 1902 Effects of potassium cyanide and of lack of oxygen upon the fertilized eggs and embryos of the sea urchin. Amer. Journ. Physiol., vol. 8 , no. 1 .

1904 Rhythms of susceptibility and of carbon dioxide production in cleavage. Amer. Journ. Physiol., vol. 11, no. 1.

E. MAUpas 1888 Recherches expérimentales sur la multiplication des infusoires ciliés. Arch. d. zool. exper. et gén., 2me ser., T. 7. 
M. Popofr 1907 Depression der Protozoenzelle und der Geschlechtszellen der Metazoen. Archiv f. Protistenkunde, R. Hertwig Festband.

J. W. Scotr, 1903 Periods of susceptibility in the differentiation of the egg of Amphitrite. Biol. Bull., vol. 5, no. 1.

E. W. Towde 1904 A study of the effects of certain stimuli, single and combined, upon Paramaecium. Amer. Journ. Physiol, vol. 12, no. 2.

L. L. WOODRUFF 1905 An experimental study on the life history of hypotrichous Infusoria. Journ. Exper. Zool., vol. 2, no. 4.

1909 Further studies on the life history of Paramaecium. Biol, Bull., vol. 17 , no. 4.

1911a Two housand generations of Paramaecium. Arohiv f. Protistenkunde, Bd. 21, 3.

$1911 b$ The effect of excretion products of Paramaecium on its rate of reproduction. Journ. Exper. Zool., vol. 10, no. 4.

L. L. Woodruff AND G. A. Battsell 1911 The reproduction of Paramaecium aurelia in a 'constant' culture medium of beef extract. Journ. Exper. Zool., vol. 11, no. 1 . 